\title{
A new formulation for two-wave Riemann solver accurate at contact interfaces.
}

\author{
Xi Deng, $1, \mathrm{a})$ Pierre Boivin, ${ }^{1, \mathrm{~b})}$ and Feng $\mathrm{Xiao}^{2, \mathrm{c})}$ \\ 1) Aix Marseille Univ, CNRS, Centrale Marseille, M2P2, Marseille, France \\ ${ }^{2)}$ Department of Mechanical Engineering, Tokyo Institute of Technology, 2-12-1(i6-29) Ookayama, Meguro-ku, \\ Tokyo, 152-8550, Japan
}

(Dated: 16 April 2019)

This study proposes a new formulation for Harten, Lax and van Leer (HLL) type Riemann solver which is capable of solving contact discontinuities accurately but with robustness for strong shock. It is well known that the original HLL, which has incomplete wave structures, is too dissipative to capture contact discontinuities accurately. On the other side, contact-capturing approximate Riemann solvers such as Harten, Lax and van Leer with Contact (HLLC) usually suffer from spurious solutions, also called carbuncle phenomenon, for strong shock. In this work a new accurate and robust HLL-type formulation, so-called HLL-BVD (HLL Riemann solver with BVD) is proposed by modifying the original HLL with BVD (boundary variation diminshing) algorithm. Instead of explicitly recovering the complete wave structures like the way of HLLC, the proposed method restores the missing contact with a jump-like function. The capability of solving contact discontinuities is further improved by minimizing the inherent dissipation term in HLL. Without modifying the original incomplete wave structures of HLL, the robustness for strong shock has been reserved. Thus the proposed method is free from shock instability problem. The accuracy and robustness of the new method are demonstrated through solving several one- and two-dimensional tests. Results indicate that the new formulation based on two-wave HLL-type Riemann solver is not only capable of capturing contact waves more accurately than the original HLL or HLLC but, most importantly, is free form carbuncle instability for strong shock.

\section{INTRODUCTION}

The modern finite volume method (FVM) for hyperbolic systems is developed based on Godunov scheme ${ }^{1}$ which assumes that (1) the flow variables within each computing elements are constant and (2) numerical fluxes are calculated from the solution of the Riemann problem. Owing to its robustness and flexibility, Godunov approach has been further developed for decades, and is popularly applied to solve Euler equations for high speed compressible flows. One branch in the development is the construction of approximate Riemann solvers, or in another word, upwind fluxes. Different from the exact Riemann solver, the approximate algorithms are noniterative and hence less expensive. Over the years, several upwind fluxes have been proposed, which can be generally categorized into flux vector splitting (FVS) and flux difference splitting (FDS) methods 2 .

The FVS methods split flux vectors into upstream and downstream travelling informations. These methods are generally less complicated and more easily compatible with implicit methods. However, they are not effective in capturing the discontinuities represented by linear waves. Thus they are prone to intensively smear out contact surfaces and shear waves. Schemes such as the StegerWarming ${ }^{3}$ and the FVS scheme of van Leer ${ }^{4}$ belong to

\footnotetext{
a) Electronic mail: deng.xi98@gmail.com Also at Department of Mechanical Engineering, Tokyo Institute of Technology

b) Electronic mail: pierre.boivin@univ-amu.fr

c) Electronic mail: xiao.f.aa@m.titech.ac.jp
}

this approach.

On the other hand, the FDS methods are constructed from approximate solution of local Riemann problem between two adjacent states. The FDS methods can be further divided into two groups as complete or incomplete Riemann solvers. The complete solvers have the same wave structures as the exact Riemann solver. For Euler equations, the wave structures comprise a contact discontinuity and two nonlinear waves which corresponds to either a shock or a rarefaction wave. By virtue of comprising all wave components, the complete FDS methods are capable of capturing linear as well as non-linear waves. This attractive quality makes complete Riemann solvers such as the Roe solver ${ }^{5}$, the Osher solver ${ }^{6}$ and Harten, Lax and van Leer with Contact (HLLC) solver widely used. Incomplete Riemann solvers, however, employ coarser wave structures which do not include the contact discontinuity. Thus, they generally bring excessive dissipation across contact. The incomplete solvers include the HLL $\underline{7}$, the Harten Lax van Leer Einfeldt (HLLE) ${ }^{8}$ and the Rusanov solver ${ }^{9}$.

Among these approximated Riemann solvers introduced above, HLLC method, which is less dissipative, is considered to be one of the most preferred FDS method because it can capture contact very accurately. Unfortunately, it may encounter a problem of numerical instability known as the carbuncle phenomenon. In fact, it has been known that Riemann solvers, which can resolve the contact and shear waves exactly, usually suffer from numerical instability in the vicinity of strong shock waves 10 . On the other side, HLL, which is more dissipative due to its incomplete wave structures, is very robust. 
It has an entropy satisfaction property, exactly resolves isolated shock and preserves positivity $\underline{7.11 .12}$ although it is notorious that it cannot resolve contact discontinuities exactly.

Consequently, there have been several attempts to construct Riemann solvers which can overcome the problem of either shock instability or exactly resolving contact discontinuities. Among these attempts, the most popular methodology is a hybrid method proposed in $\frac{13}{13}$. It is suggested that using a dissipative scheme in shock region while a less dissipative one elsewhere may tackle this problem. From then, several successful approaches $\underline{10}, \underline{14}-16$ have been proposed by relying on hybridization. However, the choice and design of switching parameters required in the hybrid methods usually have some arbitrariness. This arbitrariness in parameters may lead to failures for complex flow cases 17 . Moreover, the hybridization of complementary solvers may break the strict Rankine-Hugoniot jump conditions, which will result in smearing contact discontinuities 15 . Realizing the drawbacks of hybrid methods, other ideas have been tried to modify the HLL-type approximate Riemann solver. For example, in the work of $\frac{18}{p}$ Euler equations are split into convective and pressure parts before formulating the HLL in order to recover the contact in HLL. Through modifying the dissipation term in HLL flux, the ability to resolve the contact is further enhanced. In $\frac{17}{17}$, new HLLtype and HLLC-type Riemann solvers are constructed by modifying the shear viscosity of the original HLL and HLLC methods respectively. Although these modifications give good results in two dimensional problems, the performance has not been evaluated in one dimensional cases. Recently, several methods have been proposed to reduce shock instability for complete wave Riemann solvers through controlling numerical dissipation. In the work ${ }^{19}$, artificial viscosity has been introduced in the control equations to cure the carbuncle phenomenon. Shockstable items or shear viscosity are introduced into numerical flux in $\underline{20,21}$ to stabilize strong shock. A method named normal reconstruction procedure is proposed in ${ }^{22}$ to add dissipation to transverse velocity which tends to cause propagation of shock anomalies. $\mathrm{In}^{23,24}$, shock stability is realized by effective controlling dissipation term. Based on the fact that different reconstruction processes have different dissipation properties, the work ${ }^{25}$ alleviates shock anomalies through combing with reconstruction schemes.

Despite these efforts, there is still no consensus on how to construct an accurate and robust approximate Riemann solver. In the present work, a simple novel method, so-called HLL-BVD (HLL Riemann solver with boundary variation diminishing algorithm), is proposed to solve contact discontinuities accurately without suffering from numerical shock instability problems. By realizing that the dissipation term in HLL flux leads to the failure of accurately resolving contact, the current method makes an attempt to minimize this dissipation term through introduction of a jump-like function. In line with ${ }^{26}$, which uses Tangent of Hyperbola for INterface Capturing (THINC) function 27.28 with boundary variation diminishing (BVD) algorithm to effectively control numerical dissipation, the current work employs a jump-like THINC function to represent the information of contact discontinuities and derives a consistent BVD algorithm to minimize the dissipation term. This leads to significant improvements in the resolution of contact discontinuities. Since the new method restores the contact with an implicit algebraic way, the incomplete wave structure of HLL is preserved. Thus, unlike other methods which retrieve contact by modifying the wave structures of HLL, the robustness of HLL for strong shocks can be kept. Examined through several benchmark tests, the proposed HLL-BVD approach is shown to better represent contact interfaces and be free from shock instability problems.

The outline of the paper is as follows. In Section 2, after a brief review of HLL-type Riemann solvers, details of our HLL-BVD method are presented. In Section 3, numerical results of benchmark tests are presented and systematically compared with conventional HLL and HLLC methods. Some concluding remarks are given in Section 4.

\section{NUMERICAL METHODS}

\section{A. Governing equations}

The one-dimensional Euler equations can be expressed as

$$
\frac{\partial \mathbf{U}}{\partial t}+\frac{\partial \mathbf{F}(\mathbf{U})}{\partial x}=0
$$

under conservative form, where the physical variables vector $\mathbf{U}$ and flux vectors $\mathbf{F}(\mathbf{U})$ read

$$
\mathbf{U}=\left[\begin{array}{c}
\rho \\
\rho u \\
\rho v \\
E
\end{array}\right] \quad \text { and } \quad \mathbf{F}(\mathbf{U})=\left[\begin{array}{c}
\rho u \\
\rho u^{2}+p \\
\rho u v \\
u(E+p)
\end{array}\right]
$$

Following the conventional notation, $\rho$ denotes the density, $p$ the pressure and $E$ the total energy per unit volume. $u$ is the fluid velocity in the $x$ direction and $v$ is the transverse velocity. System (10) is closed through introduction of the perfect gas equation of state

$$
p=(\gamma-1)\left(E-\frac{1}{2} \rho\left(u^{2}+v^{2}\right)\right),
$$

where $\gamma$ is the specific heat ratio.

Within the finite volume framework, System (1) in semi-discrete form reads

$$
\frac{\partial \overline{\mathbf{U}}_{i}}{\partial t}=-\frac{1}{\Delta x}\left(\mathbf{F}_{i+\frac{1}{2}}-\mathbf{F}_{i-\frac{1}{2}}\right)
$$


where the volume-integrated average value $\overline{\mathbf{U}}_{i}$ in cell $\mathcal{I}_{i}$ is defined as

$$
\overline{\mathbf{U}}_{i} \approx \frac{1}{\Delta x} \int_{x_{i-1 / 2}}^{x_{i+1 / 2}} \mathbf{U}(x, t) d x .
$$

The numerical fluxes $\mathbf{F}_{i \pm \frac{1}{2}}$ at cell boundaries can be computed by a Riemann solver. In the following, let the subscripts $\mathbf{L}$ and $\mathbf{R}$ identify the left and right values of a given quantity on either side of the cell interface.

\section{B. HLL Riemann solvers}

Within the HLL framework, each cell boundary is regarded as a two-wave system. The system, illustrated in Fig. 1, then consists of two waves travelling at $\left(S_{L}, S_{R}\right)$.

In that context, writing the conservation equations (1) in weak form

$$
\oint \mathbf{U} \cdot d x-\mathbf{F} \cdot d t=0
$$

and assuming $\mathbf{U}$ to be constant on each branch of the integration path illustrated in Fig 1 directly leads to

$$
\mathbf{U}^{*}=\frac{S_{R} \mathbf{U}_{\mathbf{R}}-S_{L} \mathbf{U}_{\mathbf{L}}+\mathbf{F}_{\mathbf{L}}-\mathbf{F}_{\mathbf{R}}}{S_{R}-S_{L}}
$$

where the ${ }^{*}$ superscript denotes the middle state. The corresponding flux can be found as

$$
\mathbf{F}^{*}=\frac{S_{R} \mathbf{F}_{\mathbf{L}}-S_{L} \mathbf{F}_{\mathbf{R}}+S_{L} S_{R}\left(\mathbf{U}_{\mathbf{R}}-\mathbf{U}_{\mathbf{L}}\right)}{S_{R}-S_{L}} .
$$

The final flux $\mathbf{F}_{i+\frac{1}{2}}$, required to solve (3), then read

$$
\mathbf{F}_{i+\frac{1}{2}}^{h l l}= \begin{cases}\mathbf{F}_{\mathbf{L}} & \text { if } 0 \leq S_{L} \\ \mathbf{F}^{*} & \text { if } S_{L} \leq \leq S_{R} \\ \mathbf{F}_{\mathbf{R}} & \text { if } 0 \geq S_{R}\end{cases}
$$

Following 29 , the right and left wave velocities are approximated as

$S_{L}=\min \left(u_{L}-c_{L}, u_{R}-c_{R}\right), \quad S_{R}=\max \left(u_{L}+c_{L}, u_{R}+c_{R}\right)$,

where $c_{L / R}$ is the sound speed on either side of the interface.

As with most shock capturing schemes, robustness is assured by inclusion of diffusion at the shock. This can be easily seen by separating the flux (7) into two contributions

$$
\mathbf{F}^{h l l}=\frac{1}{2}\left(\mathbf{F}_{\mathbf{L}}+\mathbf{F}_{\mathbf{R}}\right)+\varepsilon_{h l l},
$$

where the first term corresponds to the classical central difference flux, and $\varepsilon_{h l l}$ can be interpreted as a numerical diffusion term

$$
\varepsilon_{h l l}=\underbrace{\frac{S_{R}+S_{L}}{2\left(S_{R}-S_{L}\right)}\left(\mathbf{F}_{\mathbf{L}}-\mathbf{F}_{\mathbf{R}}\right)}_{\varepsilon_{1}}-\underbrace{\frac{S_{L} S_{R}}{S_{R}-S_{L}}\left(\mathbf{U}_{\mathbf{L}}-\mathbf{U}_{\mathbf{R}}\right)}_{\varepsilon_{2}} .
$$

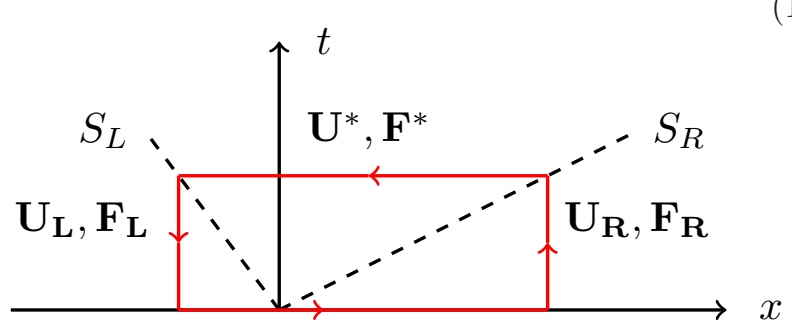

FIG. 1. The wave structure in HLL Riemann solvers. In red, the integration contour leading to Eq. (6)

When the flow presents density discontinuities, the density (and therefore $\mathbf{U}$ ) is not constant between the $S_{L}$ and $S_{R}$ waves. This leads to excessive numerical diffusion and the failure of HLL Riemann solver to accurately solve contact discontinuities ${ }^{18}$.

\section{Towards the reduction of numerical diffusion}

There are several strategies to cope with these deficiencies. One strategy, proposed in $^{18}$ is to assume local isentropy, as to replace the density jump terms in the second term of the HLL diffusion $\varepsilon_{\text {hll }}$ (11)

$$
\varepsilon_{2}=\frac{S_{L} S_{R}}{S_{R}-S_{L}}\left(\begin{array}{c}
\rho_{L}-\rho_{R} \\
(\rho u)_{L}-(\rho u)_{R} \\
(\rho v)_{L}-(\rho v)_{R} \\
E_{L}-E_{R}
\end{array}\right)
$$

into pressure jump terms

$$
\varepsilon_{2}^{i s e n}=\frac{S_{L} S_{R}}{\bar{c}^{2}\left(S_{R}-S_{L}\right)}\left(\begin{array}{c}
p_{L}-p_{R} \\
(p u)_{L}-(p u)_{R} \\
(p v)_{L}-(p v)_{R} \\
\frac{\bar{c}^{2}}{\gamma-1}\left(p_{L}-p_{R}\right)+\frac{1}{2}\left(\left(p\left(u^{2}+v^{2}\right)\right)_{L}-\left(p\left(u^{2}+v^{2}\right)\right)_{R}\right)
\end{array}\right)
$$


through introduction of an interface sound speed $\bar{c}=$ $\frac{c_{L}+c_{R}}{2}$. This method can improve the resolution across contact waves and keep shock stable. However, the isentropic hypothesis may not always hold.
A second strategy is the introduction of an antidiffusive term in (11). In that direction, the HLLEM solver ${ }^{8}$ is a possible alternative, with the diffusive term now reading

$$
\varepsilon_{\text {hllem }}=\frac{S_{R}+S_{L}}{2\left(S_{R}-S_{L}\right)}\left(\mathbf{F}_{\mathbf{L}}-\mathbf{F}_{\mathbf{R}}\right)-\frac{S_{L} S_{R}}{S_{R}-S_{L}}\left(\mathbf{U}_{\mathbf{L}}-\mathbf{U}_{\mathbf{R}}\right)+\phi \frac{S_{L} S_{R}}{S_{R}-S_{L}} M(\mathbf{U})\left(\mathbf{U}_{\mathbf{L}}-\mathbf{U}_{\mathbf{R}}\right),
$$

where the scalar $\phi \in[0,1]$ is a free parameter, and $M(\mathbf{U})$ arises from the eigen-structure of Jacobi matrix of hyperbolic system 30,31 .

Clearly, the HLLEM Riemann solver reduces to the HLL solver for $\phi=0$. Just as the HLLC solver, the HLLEM solver is flexible and able to resolve contact waves with accuracy. Unfortunately, HLLC and HLLEM solvers share similar shock instability problems, believed to be caused by perturbation transverse to the shock waves ${ }^{32}$. These comments are compatible with the observation ${ }^{33}$ that Riemann solvers explicitly dealing with the contact surface display clear evidence of carbuncle phenomenon.

This motivates the derivation of an implicit algebraic method to capture the missing contact waves in HLL Riemann solver without modifying the original two-wave system.

\section{HLL-BVD: A new formulation for HLL accurate at contact}

As stated in the previous section, the failure of HLL Riemann solver to resolve contact waves is caused by the numerical dissipation term $\varepsilon_{h l l}$ (11). Assuming constant flow variables in each cell, in particular, leads to excessive numerical dissipation due to the density jump $\left|\rho_{L}-\rho_{R}\right|$

In this work, we then suggest a procedure to minimize that contribution to the numerical dissipation $\varepsilon_{h l l}$. Inspired by ${ }^{26,34,35}$, we use the THINC function ${ }^{27,28}$ to reconstruct density jumps

$$
\tilde{\rho}_{i}(x)=\bar{\rho}_{\text {min }}+\frac{\Delta \rho}{2}\left(1+\theta \tanh \left(\beta\left(\frac{x-x_{i-1 / 2}}{x_{i+1 / 2}-x_{i-1 / 2}}-\tilde{x}_{i}\right)\right)\right)
$$

where $\bar{\rho}_{\text {min }}=\min \left(\bar{\rho}_{i-1}, \bar{\rho}_{i+1}\right), \Delta \rho=\left|\bar{\rho}_{i+1}-\bar{\rho}_{i-1}\right|$, and $\theta=\operatorname{sgn}\left(\bar{\rho}_{i+1}-\bar{\rho}_{i-1}\right)$. The jump "thickness" is controlled by parameter $\beta$, set to 1.6 throughout the validation section. It is noted that from our numerical experiments, the parameter $\beta$ valued from 1.4 to 2.0 is also able to give good or acceptable results. A smaller $\beta$ will results in more diffusive solutions while a larger one tends to introduce extra anti-diffusion effect. $\tilde{x}_{i}$ represents the position of jumps, computed as to satisfy $\bar{\rho}_{i}=\frac{1}{\Delta x} \int_{x_{i-1 / 2}}^{x_{i+1 / 2}} \tilde{\rho}_{i}(x) d x$. Having reconstructed the THINC function in every cell, it becomes straightforward to retrieve the corresponding density values $\rho_{L / R}^{T}$ on either side of the face (note the $\mathrm{T}$ superscript for THINC reconstruction).
On the other hand, we have at our disposal the interface values $\rho_{L / R}^{P}$, coming from the Polynomial reconstruction, which may vary depending on the numerical scheme. In the present work, we use MUSCL (Monotone Upstream-centered Schemes for Conservation Law) scheme ${ }^{36}$ as the polynomial reconstruction.

In order to minimize the dissipation term, a BVD algorithm which is originally proposed $\mathrm{in}^{26}$ is devised to minimize the density jumps on each cell boundaries. Final values for $\rho_{L / R}$ are picked from $\rho_{L / R}^{P}$ and $\rho_{L / R}^{T}$ values, as to minimize the density jump. In other words,

$$
\left|\rho_{L}-\rho_{R}\right|=\min \left(\left|\rho_{L}^{T}-\rho_{R}^{T}\right|,\left|\rho_{L}^{P}-\rho_{R}^{P}\right|,\left|\rho_{L}^{P}-\rho_{R}^{T}\right|,\left|\rho_{L}^{T}-\rho_{R}^{P}\right|\right),
$$

leading to a minimal density jump in the dissipative term in $\varepsilon_{h l l}$ (11), now reading

$$
\varepsilon_{\text {new }}=\frac{S_{R}+S_{L}}{2\left(S_{R}-S_{L}\right)}\left(\mathbf{F}_{\mathbf{L}}-\mathbf{F}_{\mathbf{R}}\right)-\frac{S_{L} S_{R}}{S_{R}-S_{L}}\left(\begin{array}{c}
\rho_{L}-\rho_{R} \\
\rho_{L} u_{L}-\rho_{R} u_{R} \\
\rho_{L} v_{L}-\rho_{R} v_{R} \\
\left(\frac{p_{L}}{\gamma-1}+\frac{1}{2} \rho_{L}\left(u_{L}^{2}+v_{L}^{2}\right)\right)-\left(\frac{p_{R}}{\gamma-1}+\frac{1}{2} \rho_{R}\left(u_{R}^{2}+v_{R}^{2}\right)\right)
\end{array}\right)
$$


To summarize, the numerical diffusion term of the new formulation for HLL-type Riemann solver is calculated by following steps:

1. Prepare the density values $\rho_{L / R}^{P}$ at cell interfaces by using classical Godnunov scheme or high order schemes;

2. Retrieve the discontinuous density field in the intermediate state with THINC function of (15) to get $\rho_{L / R}^{T}$;

3. Determine the intermediate density value $\rho_{L}$ and $\rho_{R}$ from $\rho_{L / R}^{T}$ and $\rho_{L / R}^{P}$ with BVD algorithm in (16) to minimize the density jump $\left|\rho_{L}-\rho_{R}\right|$ at cell boundaries;

4. The final flux is then computed through (10) and (17).

Since the contact waves are restored through an algebraic way without modifying the wave structures explicitly like HLLC or HLLEM, the numerical tests in following section will show that the new HLL-type solver can accurately solve sharp contact whilst preserving shock stability.

\section{NUMERICAL RESULTS}

This section presents a series of numerical tests conducted to evaluate the performance of the proposed new HLL-BVD Riemann solver and systematic comparison with the HLL and HLLC solvers are presented. Throughout this section, the specific heat ratio is set as $\gamma=1.4$. The CFL number is set to 0.4 unless specifically noted.

\section{A. Isolated contact}

Let us first consider contact wave propagation at low Mach numbers. A one-dimensional domain is initialized as

$$
\left(\rho_{0}, u_{0}, p_{0}\right)=\left\{\begin{array}{ll}
(1.4,0.1,1.0) & 0 \leq x \leq 0.5 \\
(1.0,0.1,1.0) & \text { otherwise }
\end{array},\right.
$$

corresponding to a contact discontinuity moving at $M a=$ 0.1. The computation is carried out with 100 uniform cells and with time integrated up to $t=2.0$. Figure 2] a presents the density profile as obtained through integration with the classical HLL and HLLC solvers, as well as the present HLL-BVD solver. It can be seen that the moving contact wave resolved by the original HLL Riemann solver is the most diffusive. Although HLLC can resolve the moving contact wave better than HLL, it still poorly represents the contact wave as a sharp discontinuity. However, the proposed HLL-BVD has the ability to capture the sharp jump of density field across the contact. The test shows that the proposed HLL-BVD is superior

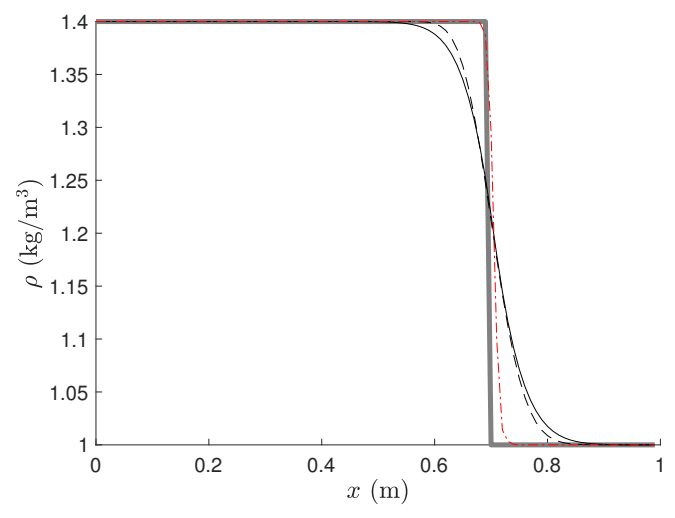

(a) $\mathrm{Mach}=0.1$

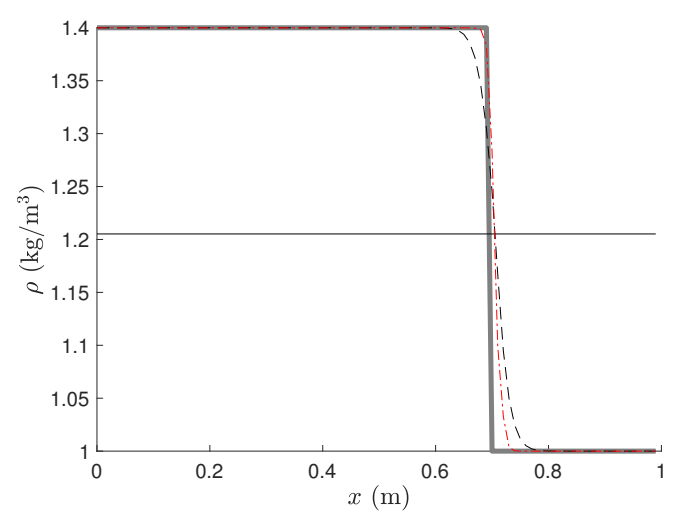

(b) $\mathrm{Mach}=10^{-5}$

FIG. 2. Density profiles for an isolated right-moving contact wave travelling at Mach $=0.1$ (a) and Mach $=10^{-5}$ (b), as obtained with the HLL (solid), HLLC (dashed) and present (dot-dashed) solvers. The reference solution is represented by the thick line.

to original HLL and HLLC regarding to represent sharp contact discontinuities.

To further examine the performance of the proposed formulation, the same test case is carried out at a very low Mach Number, $M a=10^{-5}$. The results, reported in Fig. 2 b, exhibit the failure of the HLL solver, consistently with previous reports $37-39$. Our HLL-BVD proposal, however, successfully capture the contact discontinuity, even at very low Mach number.

\section{B. Sod's problem}

Our second test case is the Sod problem, with initial conditions set as 40

$$
\left(\rho_{0}, u_{0}, p_{0}\right)= \begin{cases}(1,0,1) & 0 \leq x \leq 0.5 \\ (0.125,0,0.1) & \text { otherwise }\end{cases}
$$

This initial condition will result in a right running shock, a right running contact wave and a left running rarefaction wave. With the same number of cells as in previous 


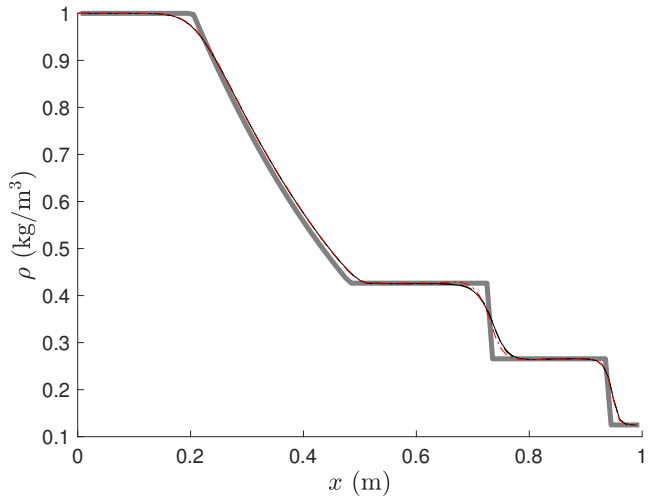

(a) Density

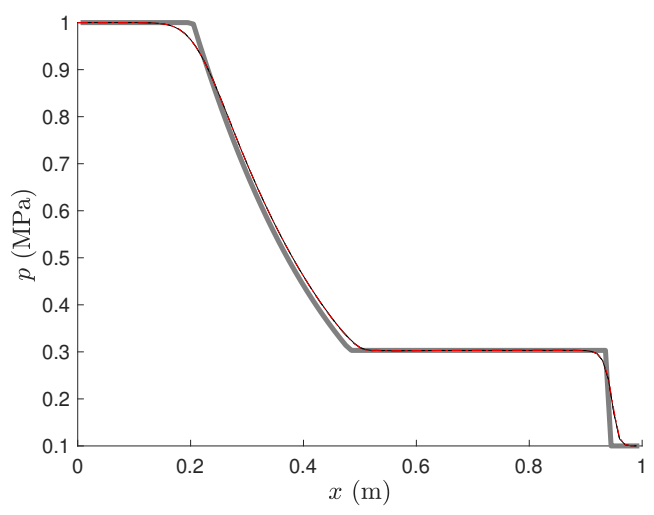

(b) Pressure

FIG. 3. Density (a) and pressure (b) profiles for Sod problem with 100 cell elements, as obtained with the HLL (solid), HLLC (dashed) and present (dot-dashed) solvers. The reference solution is represented by the thick line.

case, the computation lasts until time $t=0.25$. The density and pressure profiles, displayed in Fig 3 , are very similar whether the HLL, HLLC or HLL-BVD solver is used, with the exception of the contact discontinuity, slightly thinner in the HLL-BVD case.

\section{Lax's problem}

The Lax problem is employed here to check the performance of the proposed numerical scheme to capture relatively strong shock ${ }^{41}$. The initial condition is

$$
\left(\rho_{0}, u_{0}, p_{0}\right)=\left\{\begin{array}{ll}
(0.445,0.698,3.528) & 0 \leq x \leq 0.5 \\
(0.5,0,0.571) & \text { otherwise }
\end{array} .\right.
$$

A relatively strong right running shock, a right running contact discontinuity and a left running expansion wave will evolve with this initial condition. Numerical solutions for density and pressure field are presented in Fig. 4. Again, solutions for expansion fan and shock are almost identical for all three types of HLL Riemann solvers.

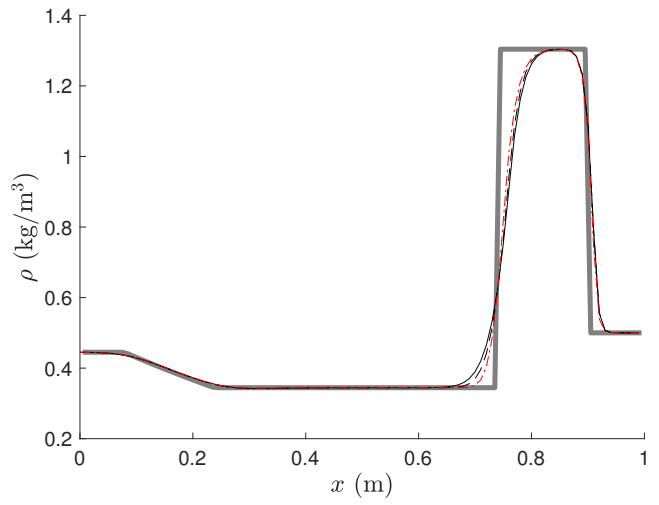

(a) Density

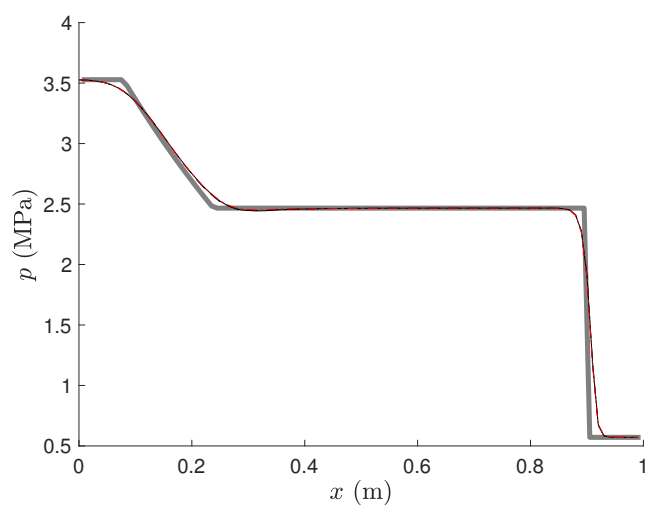

(b) Pressure

FIG. 4. Density (a) and pressure (b) profiles for Lax problem with 100 cell elements, as obtained with the HLL (solid), HLLC (dashed) and present (dot-dashed) solvers. The reference solution is represented by the thick line.

Again, HLL-BVD solves the contact discontinuity with slightly less numerical diffusion.

\section{Strong Lax problem}

In this test, a numerical test involving a strong shock is considered here to examine the ability of numerical methods to capture contact discontinuities as well as strong shock waves. The initial condition is set as following:

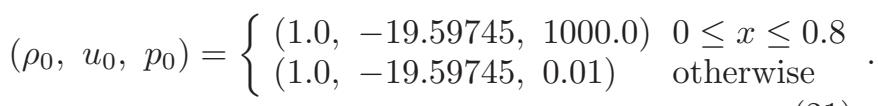

The solution consists of a strong shock, a stationary contact and a left running expansion wave. The numerical results at $t=0.012$ from different Riemann solvers are presented in Fig. 5 for density field and Fig. 6 for pressure field. For this case, it seems that the HLLC Riemann solver can resolve stationary contact with the least amount of numerical diffusion. However, it produces the largest overshoot values across the shock waves as shown 


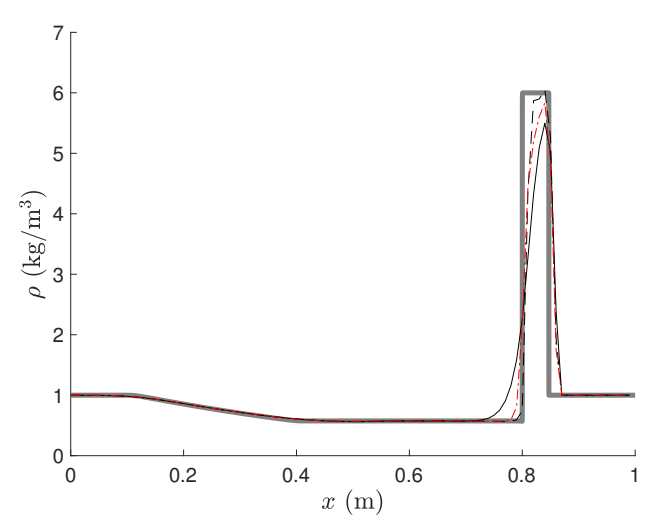

(a) Density

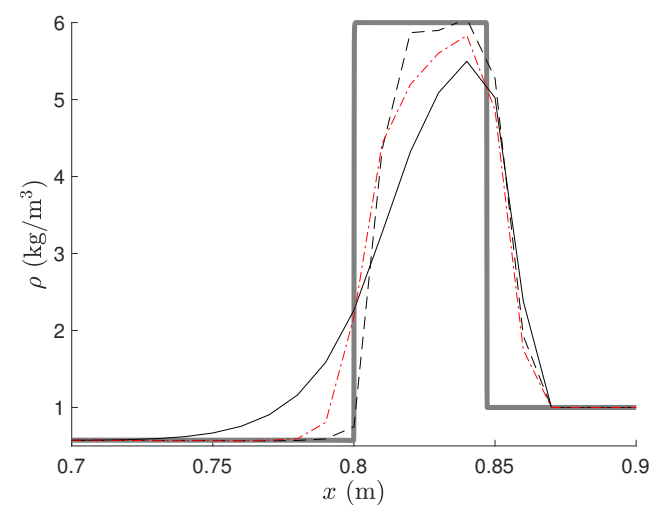

(b) Density field in zoomed region

FIG. 5. Global (a) and magnified (b) density profiles for strong Lax problem, as obtained with the HLL (solid), HLLC (dashed) and present (dot-dashed) solvers. The reference solution is represented by the thick line.

in zoomed region of Fig. [5] and Fig. [6] which may lead unstable solutions for strong shock. However, the proposed one has the ability to represent stationary contact with much less numerical diffusion than the original HLL but also capture strong shock waves without overshooting.

\section{Slow moving shock}

To further investigate the abilities of different HLLtype Riemann solvers to capture shock waves stably, the slowly moving shock problem is considered here. The computational domain is still one unit long, but now consists of 1000 elements. The initial condition of slowly right moving shock is set as follows

$$
\left(\rho_{0}, u_{0}, p_{0}\right)=\left\{\begin{array}{ll}
(1.0,-3.44,1.0) & 0 \leq x \leq 0.5 \\
(3.86,-0.81,10.33) & \text { otherwise }
\end{array} .\right.
$$

The post-shock condition is set at the left boundary while the pre-shock condition is set at the right side. The numerical solutions are obtained at $t=1.5$ and shown in

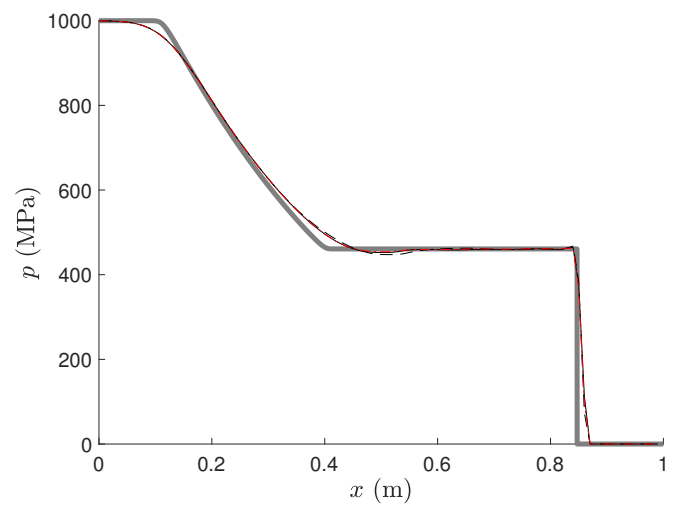

(a) Pressure

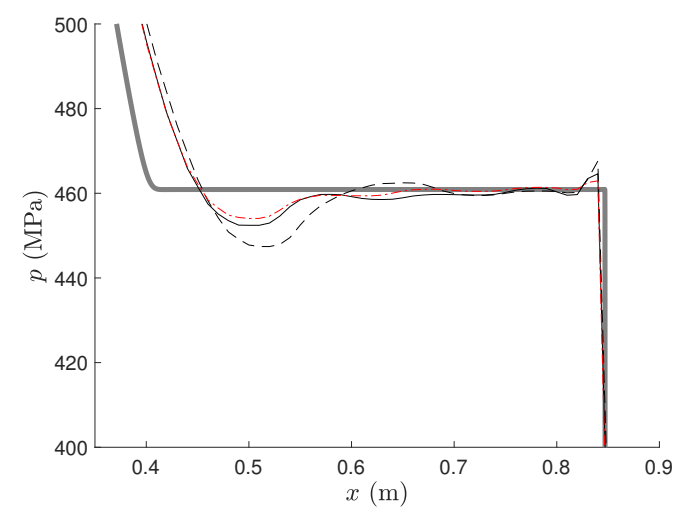

(b) Pressure field in zoomed region

FIG. 6. Global (a) and magnified (b) pressure profiles for strong Lax problem, as obtained with the HLL (solid), HLLC (dashed) and present (dot-dashed) solvers. The reference solution is represented by the thick line.

Fig. 7. Among the three solvers, HLLC leads to the maximum oscillation amplitude in the post-shock state, while HLL-BVD clearly leads to the most stable solution. As shown in two-dimensional tests later, the unstable shock waves from HLLC may result in unacceptable solutions.

\section{E. 2D Riemann problems}

Let us consider two-dimensional problem, starting with the two-dimensional Riemann problem proposed and extensively studied in $n^{42,43}$. It has been reported in $\underline{44}$ that sharp capturing of discontinuities as well as shear waves is critical to capture the small-scale structures created by the Kelvin-Helmholtz instability.

The computational domain is $[-0.5,0.5] \times[-0.5,0.5]$. Two different configurations are studied in the present work. The initial conditions are set as follows, 


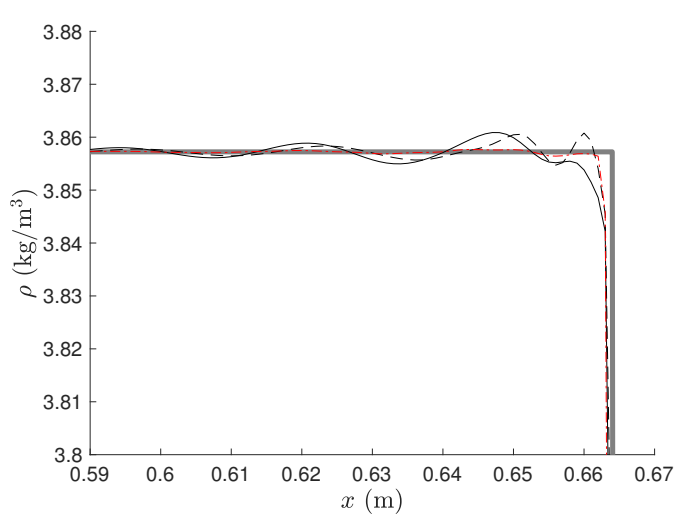

(a) Density

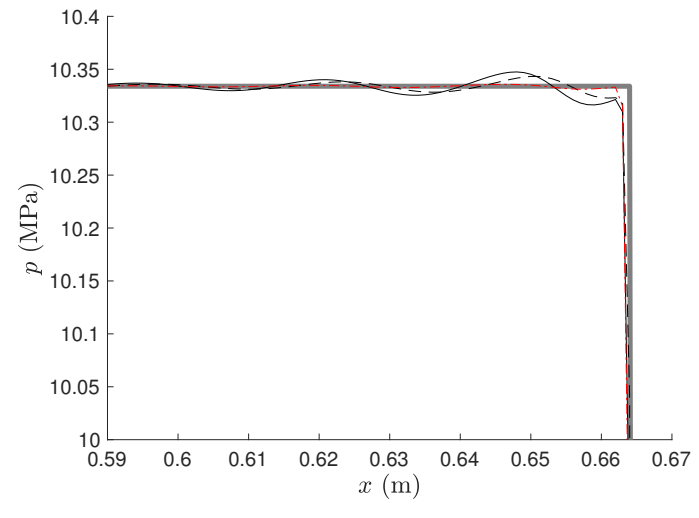

(b) Pressure

FIG. 7. Density (a) and pressure (b) profiles for the slow moving shock problem, as obtained with the HLL (solid), HLLC (dashed) and present (dot-dashed) solvers. The reference solution is represented by the thick line.

$$
\text { Case 1: } \quad\left(\rho_{0}, u_{0}, v_{0}, p_{0}\right)= \begin{cases}(2.0,0.75,0.5,1.0) & x \leq 0.0, y \geq 0.0 \\ (1.0,-0.75,0.5,1.0) & x<0.0, y<0.0 \\ (1.0,0.75,-0.5,1.0) & x>0.0, y>0.0 \\ (3.0,-0.75,-0.5,1.0) & x>0.0, y<0.0\end{cases}
$$

Uniform grids of $600 \times 600$ are employed in our calculation. The numerical results calculated from different HLL-type Riemann solvers are presented in Fig. 8 9. These results clearly indicate the superiority of our HLL-BVD solver over the classical HLL and HLLC options in capturing contact and shear waves. The solutions produced by the original HLL Riemann solver are the most diffusive, which will lead to failure of resolving Kelvin-Helmholtz instability. The tests in this subsection demonstrate that the proposed HLL-BVD can work well in two dimensional problems whilst significantly improving the capability to solve contact discontinuities and associated fine flow structures.

\section{F. Odd-even perturbation problem}

It is well known that Riemann solvers which can accurately capture contact discontinuities usually suffer from spurious solutions, called carbuncle instabilities, in the vicinity of strong shock. Solving strong shock is especially important for problems involving reaction and heat release $\underline{45} \underline{\underline{48}}$. In this case, the odd-even decoupling test originally proposed in 13 is employed to examine the ability of the proposed HLL-BVD to obtain the stability of strong shocks. The computational domain consists of $51 \times 26$ elements. Initially, a steady normal shock of Mach $=20$ is set in the domain with initial conditions for left $(i \leq 39)$ and right $(i \geq 41)$ sides following RankineHugoniot conditions. An intermediate state is prescribed at $i=40$ where the internal shock conditions are given as $\rho_{M}=\lambda \rho_{L}+(1-\lambda) \rho_{R}$ with other variables calculated based on $\rho_{M}$. A perturbation of shock along y-direction is produced by assigning different $\lambda$ as

$$
\lambda=\left\{\begin{array}{ll}
0.2 \text { if } j \text { is odd } \\
0.4 \text { if } j \text { is even }
\end{array} .\right.
$$

The initial density contour of odd-even perturbation is presented in the first plot of Fig. 10. The $\mathrm{CFL}=0.5$ is used. We examine the solutions from different Riemann solvers. The contour plots after $1 \times 10^{4}$ time steps and $4 \times 10^{4}$ time steps are presented in Fig. 10. The density distributions along $\mathrm{x}$-direction in the middle of domain are also plotted in the Fig. 11. From these results, oddeven decoupling is clearly visible with HLLC, with which 


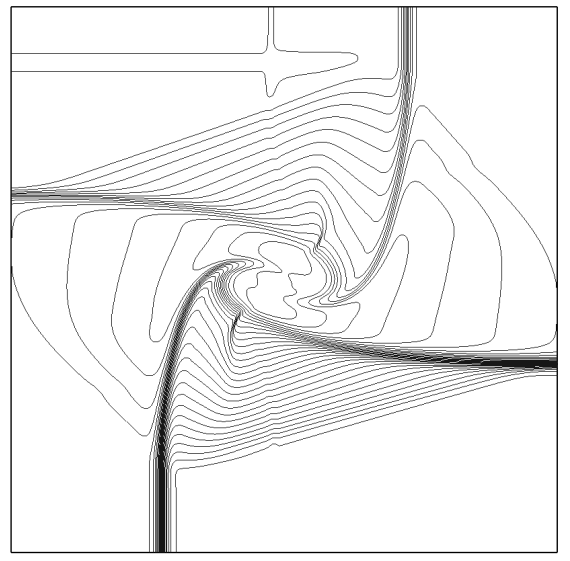

(a) HLL

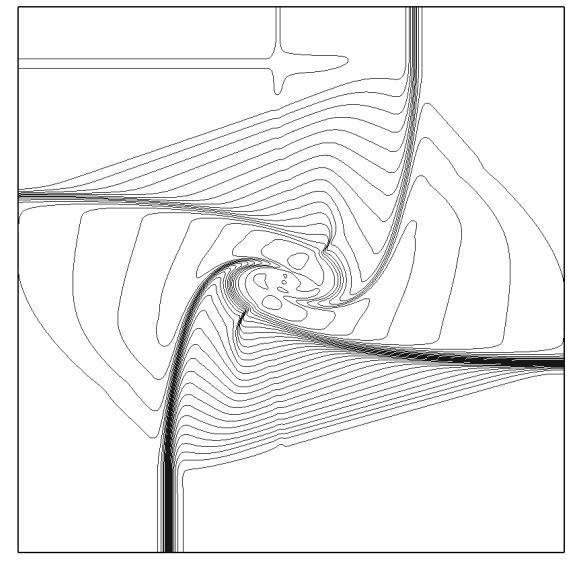

(b) HLLC

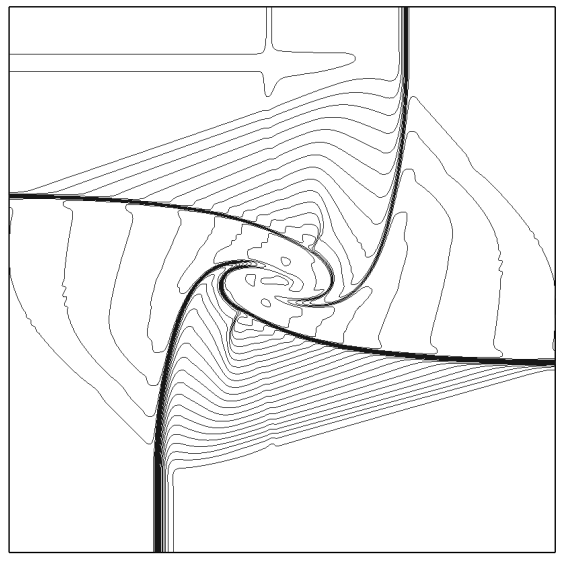

(c) HLL-BVD

FIG. 8. Density contour for 2D Riemann problems with Case 1 initial condition. Solutions using uniform grids of $600 \times 600$ from different formulation for HLL-type Riemann solvers are presented.

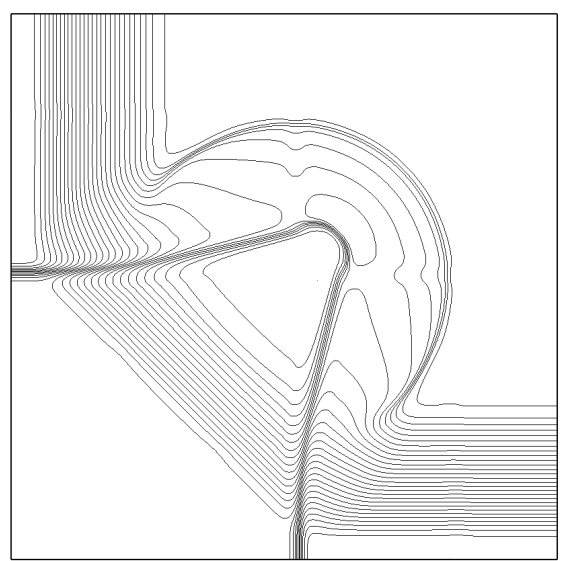

(a) HLL

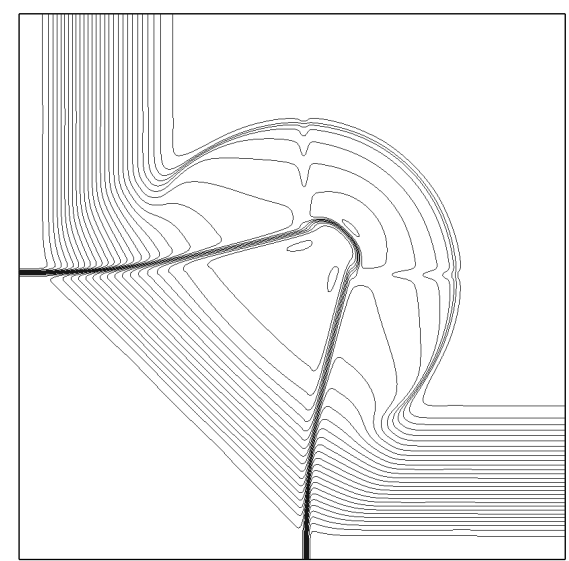

(b) HLLC

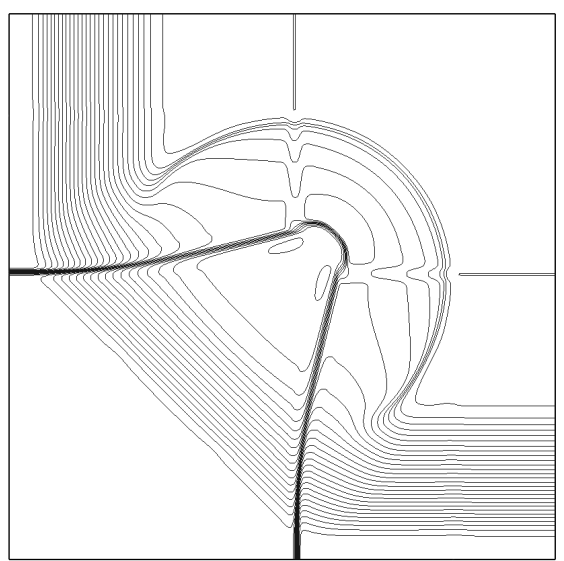

(c) HLL-BVD

FIG. 9. Density contour for 2D Riemann problems with Case 2 initial condition. Solutions using uniform grids of $600 \times 600$ from different HLL-type Riemann solvers are presented.

original steady shock suffers from instabilities and dies out after $4 \times 10^{4}$ time steps. Whereas, HLL and proposed one are free from carbuncle instability. The test shows that the proposed formulation is more robust than HLLC when solving strong shocks.

\section{G. Sedov blast wave problem}

A spherically symmetric explosion, known as Sedov blast wave problem, is considered here. The computational domain is $[-1.1,1.1] \times[-1.1,1.1]$ with 160 grid elements in each direction. The initial conditions are given by $\left(\rho_{0}, u_{0}, v_{0}, p_{0}\right)=\left(1.0,0.0,0.0,10^{-6}\right)$ except in the central cell where an initial delta-function energy source was set in terms of pressure as

$$
p_{c}=(\gamma-1) \rho_{0} \frac{\epsilon_{0}}{V_{c}}
$$

in which $V_{c}$ denotes the volume of the cell containing the origin energy source and $\epsilon_{0}$ is the total amount of released energy. In our numerical tests, the released energy is $\epsilon_{0}=0.244816$. The simulations are carried out up to time $t=1.0$ at which the shock front reaches a radius of $R=1$. Fig. 12 shows the numerical results obtained by the HLL, HLLC and the proposed method. As one can see, the results from the HLLC exhibit carbuncle instability across the shock front. Whereas, both HLL and the proposed method are capable of solving stable shock front. To make a quantitative comparison, we plot numerical solutions with different solvers and exact solution ${ }^{49-51}$ in Fig. 13. It is obvious that HLLC produces the largest numerical errors due to carbuncle 


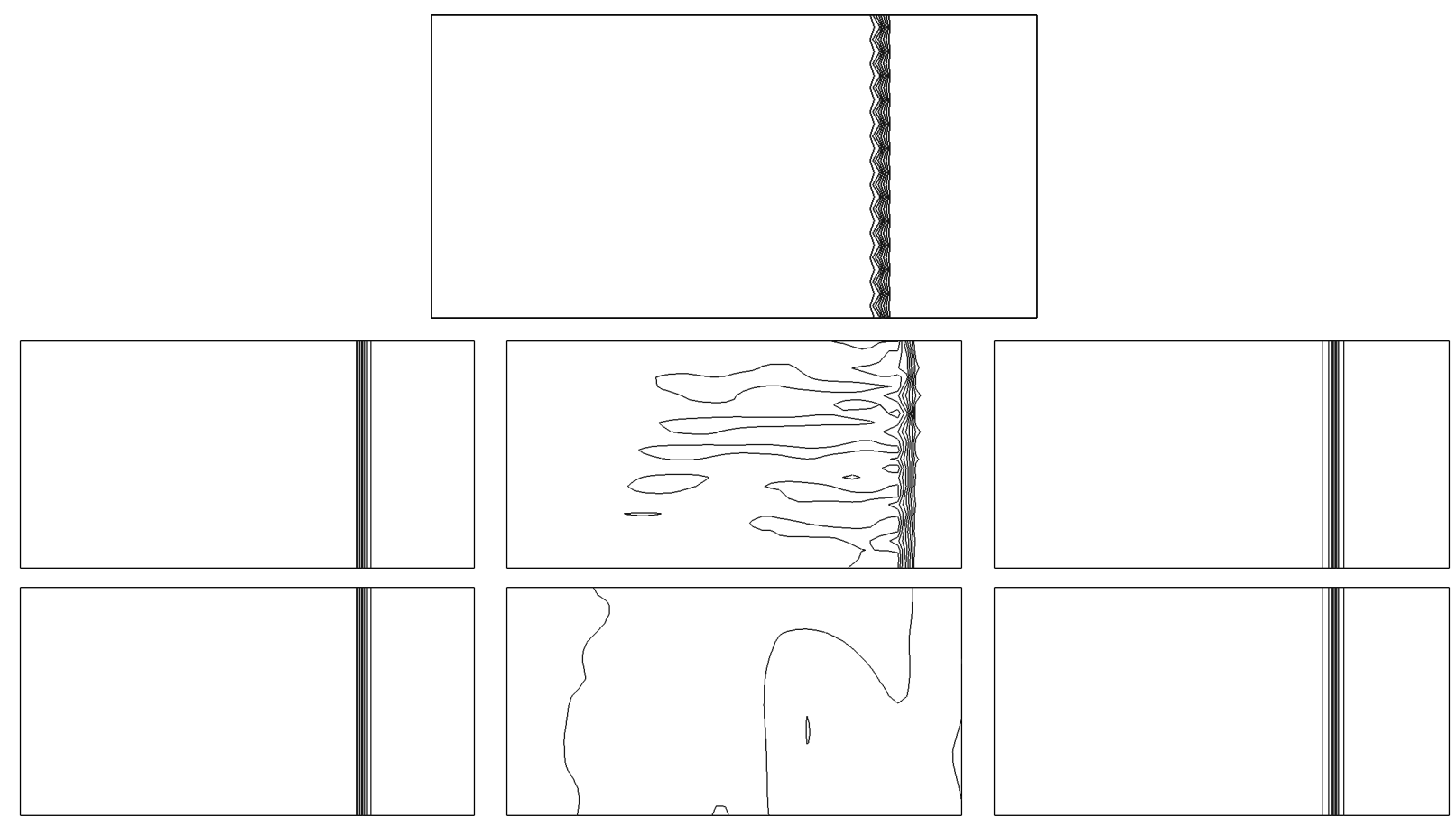

FIG. 10. Density contours for the odd-even perturbation problem as obtained with the HLL (left), HLLC (middle) and HLL-BVD (right) solvers. Initial condition (top), after 10000 (middle) and 40000 time steps.

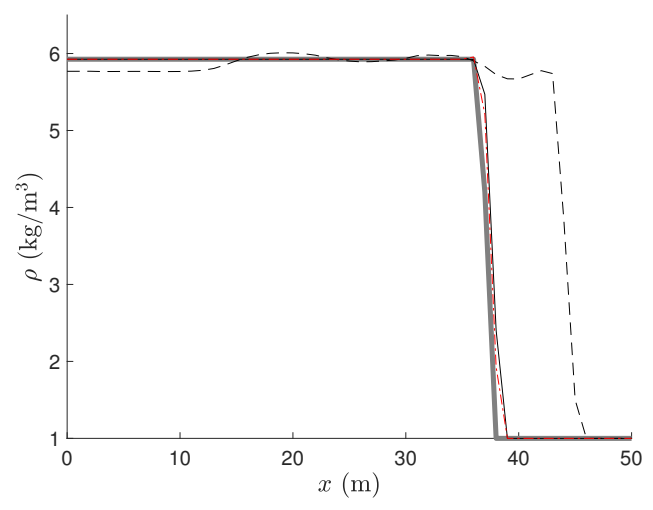

(a) 10000 time steps

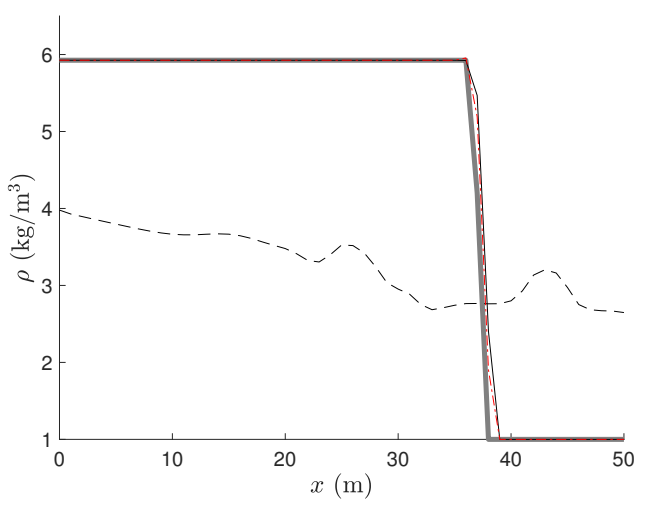

(b) 40000 time steps

FIG. 11. Density distribution along x-direction in the middle of domain for the odd-even perturbation problem (Fig. 10), as obtained with the HLL (solid), HLLC (dashed) and present (dot-dashed) solvers. The reference solution is represented by the thick line. Numerical solutions after 10000 time steps are shown in (a), while after 40000 time steps are presented in (b).

issue.

\section{H. Double Mach reflection}

To further examine that the proposed HLL-type Riemann solver is free from carbuncle phenomenon, a $M a=$ 10 hypersonic propagating planar shock reflected by $30^{\circ}$ ramp is simulated in this case. It is well known that the kinked Mach stem may occur due to carbuncle instability from Riemann solvers. This problem is proposed in 52 . The computational domain is $[0,3.2] \times[0,1]$. A right-moving Mach 10 shock is imposed with $60^{\circ}$ angle relative to $x$-axis. At the right boundary of the computational domain, the boundary condition is given by setting all gradients to be zero. The solution is computed up to $t=0.2$ with different Riemann solvers. The results are displayed in Fig. 14. It is clearly visible that kinked Mach stem occurs in case of HLLC, which gives rise to spurious triple point and polluted region after shock. Whereas the spurious kinked Mach stem is completely eliminated with HLL or the proposed one. Moreover, the roll-up region 


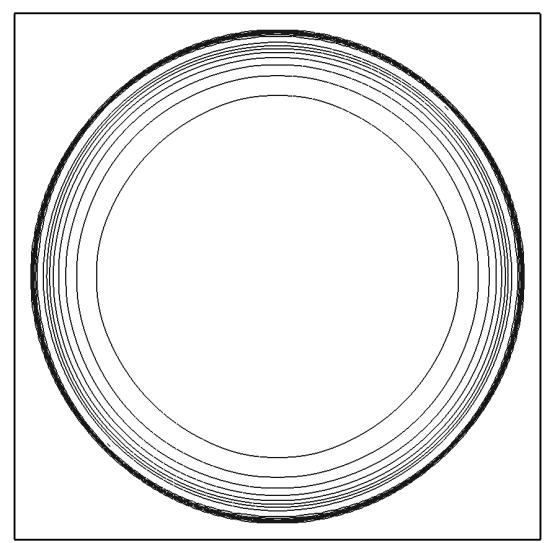

(a) HLL

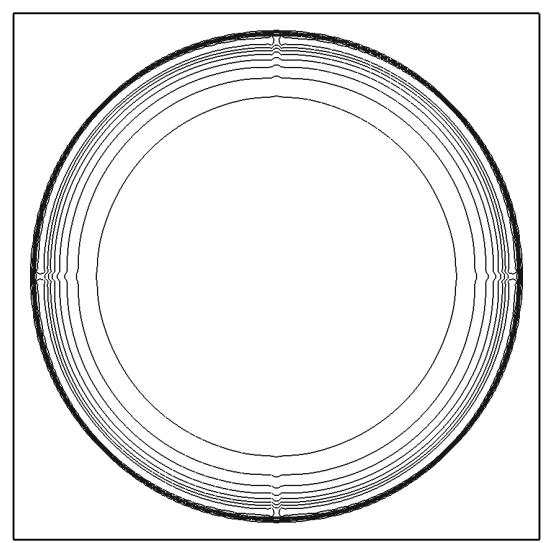

(b) HLLC

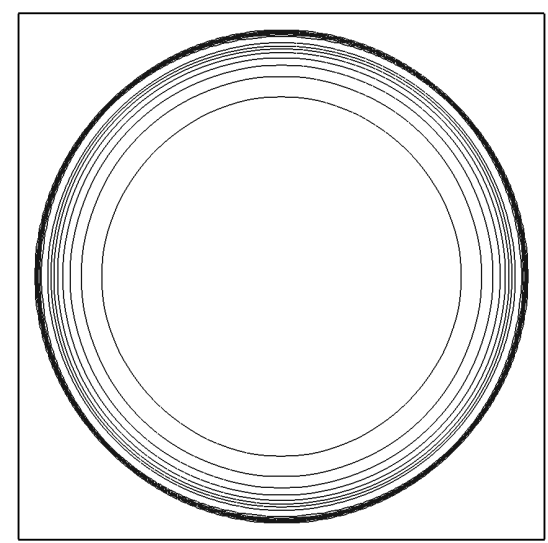

(c) HLL-BVD

FIG. 12. Density contour calculated by different Riemann solvers until $t=1.0$ for the Sedov blast wave problem. It is noted that in the $\mathrm{x}$ - and $\mathrm{y}$-direction, some numerical oscillations caused by the carbuncle instability can be observed for HLLC.

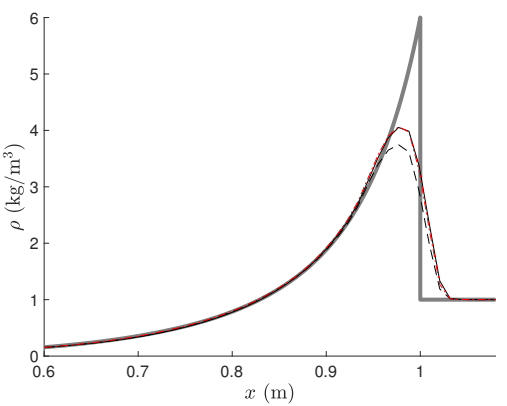

FIG. 13. The radial distribution of the density field for the the Sedov blast wave problem , as obtained with the HLL (solid), HLLC (dashed) and present (dot-dashed) solvers. The reference solution is represented by the thick line.

around slip line is solved with less numerical diffusion by the new formulation compared with the original HLL.

\section{Rayleigh-Taylor instability}

Let us conclude the validation section with a simulation of the Rayleigh-Taylor instability problem 53,54 . Initially, a single contact discontinuity separating two fluids with different densities is set at $y=0.0$. A small perturbation is given to the velocity. Due to the density difference, the heavy fluid will accelerate into the light one. The Rayleigh-Taylor instability will develop as the spike of the heavy fluid falls into the light one and the bubble of the light fluid rises into the ambient heavy fluid. The computation domain is $[0,0.25] \times[0,1]$. The initial condition is given by

$\left(\rho_{0}, u_{0}, v_{0}, p_{0}\right)=\left\{\begin{array}{l}(2,0,-\tau \cos (8 \pi x), 1+2 y) 0 \leq y \leq 0.5 \\ (1,0,-\tau \cos (8 \pi x), 1.5+y) \text { otherwise }\end{array}\right.$ where $\tau=0.025 \sqrt{\gamma p / \rho}$, and the specific heat is chosen to be $\gamma=5 / 3$ in this case. To model the effect of density difference, a source term $\rho$ is added to the right hand of the momentum equations in the $\mathrm{y}$-direction and a source term $\rho v$ is added to the energy equation. The computation is carried out up to the $t=1.95 \mathrm{~s}$ with $60 \times 240$ cell elements. As studied in 34,53 , low-dissipation methods are essential to resolve the sharp contact and associated small scale structures due to Rayleigh-Taylor instability. We plot the density contour calculated by different Riemann solvers in the left panel of Fig. 15. Clearly HLL is the most diffusive solver and can not resolve sharp contact. Both HLLC and proposed HLLBVD methods produce high resolution results. The proposed HLL-BVD method obtains sharper material interfaces compared with HLLC. To evaluate the numerical solution quantitatively, we plot the temporal evolution of the spike and bubble tip in the right panel of Fig. 15. As studied in 54 , numerical solvers with excessive errors are not able to correctly predict the temporal evolution of instability. In our results, we find that HLL underestimates the evolution of bubble and spike characteristics due to excessive numerical dissipation errors. And the HLL and HLL-BVD produce very similar prediction.

\section{CONCLUDING REMARKS}

This work proposes a novel formulation called HLLBVD which is simultaneously capable of solving contact discontinuities accurately and of being free from shock instability. The missing contact in original HLL is restored with a jump-like THINC function. The resolution of contact wave is further improved by minimizing the dissipation term in HLL. Without modifying the waves structure explicitly, the robustness of original HLL has been preserved, as shown through one-dimensional and two-dimensional benchmark test cases. The new formulation significantly improves the resolution of contact wave 


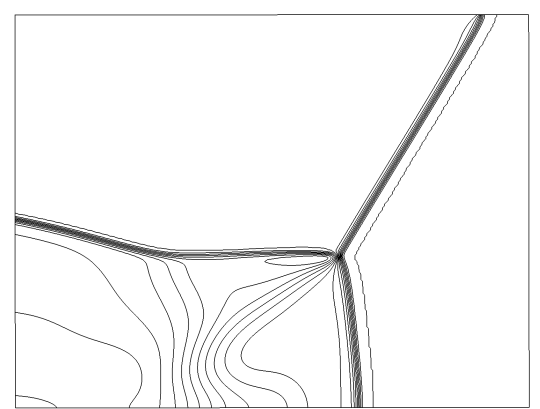

(a) HLL

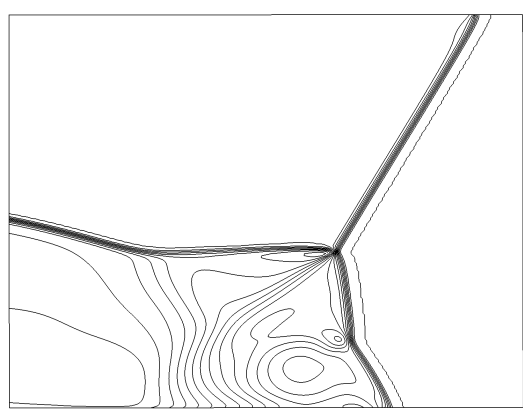

(b) HLLC

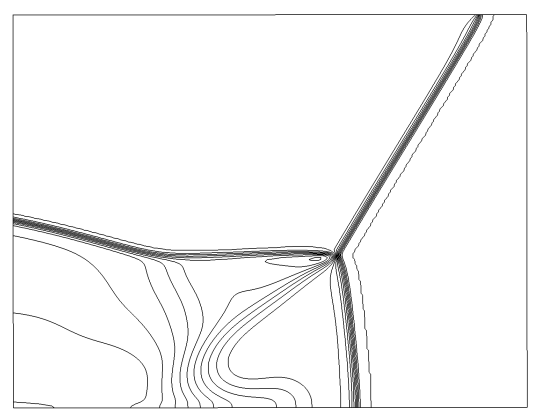

(c) HLL-BVD

FIG. 14. Density contours for double Mach reflection at $t=0.2$ with different Riemann solvers.
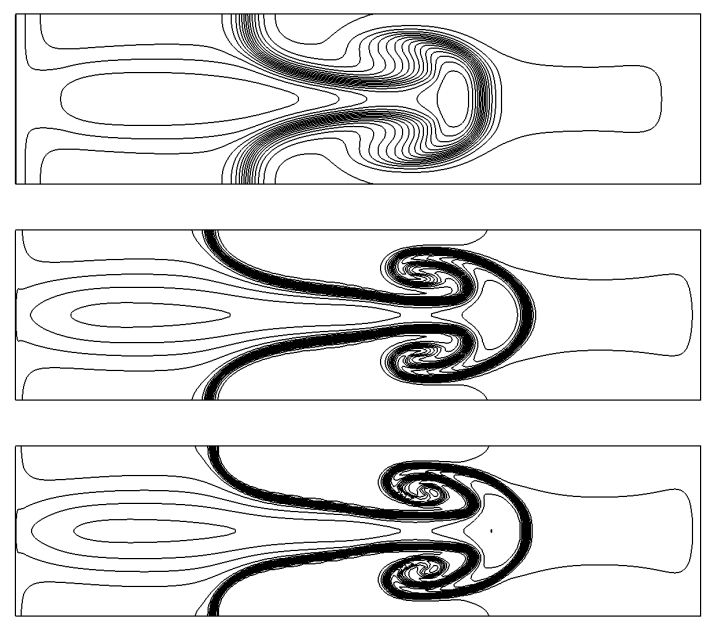

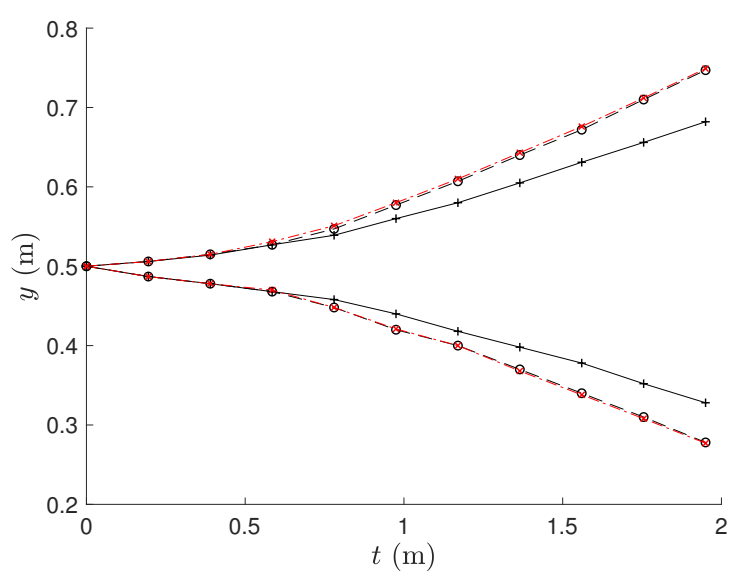

FIG. 15. Density contours for the Rayleigh-Taylor instability problem at $t=1.95 \mathrm{~s}$ with different Riemann solvers are shown in the left panel (HLL, HLLC and HLL-BVD from top to bottom). In the right panel, we show the temporal variation of bubble and spike tip positions for the Rayleigh-Taylor instability problems obtained with the HLL (solid), HLLC (dashed) and present (dot-dashed) solvers. Results from the HLLC and HLL-BVD solvers are practically indistinguishable.

and, most importantly, is free from carbuncle instability for strong shock.

Future work will investigate whether the reduced dissipation across the contact interface also improves the behavior of the HLL solver for the description of viscous flow, often penalized by excessive dissipation across the shear wave $\frac{16,20}{6}$.

\section{ACKNOWLEDGMENT}

This work was supported in part by the fund from JSPS (Japan Society for the Promotion of Science) under Grant Nos. 17K18838 and 18H01366. We also acknowledge support from Labex MEC (ANR-10-LABX0092) and the A*MIDEX project (ANR-11-IDEX-000102), funded by the "Investissements d'Avenir", for funding Xi Deng at M2P2. 
${ }^{1}$ S. K. Godunov, A difference method for numerical calculation of discontinuous solutions of the equations of hydrodynamics, Matematicheskii Sbornik 89 (3) (1959) 271-306.

${ }^{2}$ R. J. LeVeque, Finite volume methods for hyperbolic problems, Vol. 31, Cambridge university press, 2002.

${ }^{3}$ J. L. Steger, R. Warming, Flux vector splitting of the inviscid gasdynamic equations with application to finite-difference methods, Journal of computational physics 40 (2) (1981) 263-293.

${ }^{4}$ B. Van Leer, Flux-vector splitting for the euler equation, in: Upwind and High-Resolution Schemes, Springer, 1997, pp. 80-89.

${ }^{5}$ P. L. Roe, Approximate riemann solvers, parameter vectors, and difference schemes, Journal of computational physics 43 (2) (1981) 357-372.

${ }^{6} \mathrm{~S}$. Osher, F. Solomon, Upwind difference schemes for hyperbolic systems of conservation laws, Mathematics of computation 38 (158) (1982) 339-374.

${ }^{7}$ A. Harten, P. D. Lax, B. v. Leer, On upstream differencing and godunov-type schemes for hyperbolic conservation laws, SIAM review 25 (1) (1983) 35-61.

${ }^{8}$ B. Einfeldt, On godunov-type methods for gas dynamics, SIAM Journal on Numerical Analysis 25 (2) (1988) 294-318.

${ }^{9} \mathrm{~V}$. V. Rusanov, Calculation of interaction of non-steady shock waves with obstacles, NRC, Division of Mechanical Engineering, 1962.

${ }^{10}$ S. D. Kim, B. J. Lee, H. J. Lee, I.-S. Jeung, Robust hllc riemann solver with weighted average flux scheme for strong shock, Journal of Computational Physics 228 (20) (2009) 7634-7642.

${ }^{11} \mathrm{E}$. F. Toro, Riemann solvers and numerical methods for fluid dynamics: a practical introduction, Springer Science \& Business Media, 2013.

${ }^{12}$ B. Einfeldt, C.-D. Munz, P. L. Roe, B. Sjögreen, On godunovtype methods near low densities, Journal of computational physics 92 (2) (1991) 273-295.

13 J. J. Quirk, A contribution to the great riemann solver debate, in: Upwind and High-Resolution Schemes, Springer, 1997, pp. 550-569.

${ }^{14}$ K. Huang, H. Wu, H. Yu, D. Yan, Cures for numerical shock instability in hllc solver, International journal for numerical methods in fluids 65 (9) (2011) 1026-1038.

${ }^{15} \mathrm{H}$. Wu, L. Shen, Z. Shen, A hybrid numerical method to cure numerical shock instability, Commun. Comput. Phys. 8 (2010) $1264-1271$

${ }^{16}$ H. Nishikawa, K. Kitamura, Very simple, carbuncle-free, boundary-layer-resolving, rotated-hybrid riemann solvers, Journal of Computational Physics 227 (4) (2008) 2560-2581.

${ }^{17}$ Z. Shen, W. Yan, G. Yuan, A robust hllc-type riemann solver for strong shock, Journal of Computational Physics 309 (2016) 185-206.

${ }^{18}$ J. Mandal, V. Panwar, Robust hll-type riemann solver capable of resolving contact discontinuity, Computers \& Fluids 63 (2012) $148-164$.

${ }^{19} \mathrm{~A}$. V. Rodionov, Artificial viscosity in godunov-type schemes to cure the carbuncle phenomenon, Journal of Computational Physics 345 (2017) 308-329.

${ }^{20}$ S. Chen, C. Yan, B. Lin, L. Liu, J. Yu, Affordable shock-stable item for godunov-type schemes against carbuncle phenomenon, Journal of Computational Physics 373 (2018) 662-672.

${ }^{21} \mathrm{~S}$. Chen, C. Yan, B. Lin, Y. Li, A new robust carbuncle-free roe scheme for strong shock, Journal of Scientific Computing 77 (2) (2018) 1250-1277.

${ }^{22}$ Z. Chen, X. Huang, Y. Ren, M. Zhou, General procedure for riemann solver to eliminate carbuncle and shock instability, AIAA Journal 55 (6) (2017) 2002-2015.

${ }^{23} \mathrm{~S}$. Simon, J. Mandal, A cure for numerical shock instability in hllc riemann solver using antidiffusion control, Computers \& Fluids 174 (2018) 144-166.

${ }^{24}$ S. Simon, J. Mandal, A simple cure for numerical shock instability in the hllc riemann solver, Journal of Computational Physics 378 (2019) 477-496.
${ }^{25}$ Z. Jiang, C. Yan, J. Yu, B. Gao, Effective technique to improve shock anomalies and heating prediction for hypersonic flows, AIAA Journal (2017) 1475-1479.

${ }^{26}$ Z. Sun, S. Inaba, F. Xiao, Boundary variation diminishing (bvd) reconstruction: A new approach to improve godunov schemes, Journal of Computational Physics 322 (2016) 309-325.

${ }^{27}$ F. Xiao, S. Ii, C. Chen, Revisit to the thinc scheme: a simple algebraic vof algorithm, Journal of Computational Physics 230 (19) (2011) 7086-7092.

${ }^{28}$ F. Xiao, Y. Honma, T. Kono, A simple algebraic interface capturing scheme using hyperbolic tangent function, International Journal for Numerical Methods in Fluids 48 (9) (2005) 10231040.

${ }^{29}$ S. Davis, Simplified second-order godunov-type methods, SIAM Journal on Scientific and Statistical Computing 9 (3) (1988) 445473.

${ }^{30}$ D. S. Balsara, Self-adjusting, positivity preserving high order schemes for hydrodynamics and magnetohydrodynamics, Journal of Computational Physics 231 (22) (2012) 7504-7517.

${ }^{31}$ M. Dumbser, D. S. Balsara, A new efficient formulation of the hllem riemann solver for general conservative and nonconservative hyperbolic systems, Journal of Computational Physics 304 (2016) 275-319.

${ }^{32}$ L. Flandrin, P. Charrier, An improved approximate riemann solver for hypersonic bidimensional flows, in: Fourteenth International Conference on Numerical Methods in Fluid Dynamics, Springer, 1995, pp. 251-258.

${ }^{33}$ M. Pandolfi, D. D'Ambrosio, Numerical instabilities in upwind methods: analysis and cures for the "carbuncle" phenomenon, Journal of Computational Physics 166 (2) (2001) 271-301.

${ }^{34}$ X. Deng, B. Xie, R. Loubère, Y. Shimizu, F. Xiao, Limiter-free discontinuity-capturing scheme for compressible gas dynamics with reactive fronts, Computers \& Fluids 171 (2018) 1-14.

${ }^{35}$ X. Deng, S. Inaba, B. Xie, K.-M. Shyue, F. Xiao, High fidelity discontinuity-resolving reconstruction for compressible multiphase flows with moving interfaces, Journal of Computational Physics 371 (2018) 945-966.

${ }^{36} \mathrm{~B}$. Van Leer, Towards the ultimate conservative difference scheme. v. a second-order sequel to godunov's method, Journal of computational Physics 32 (1) (1979) 101-136.

${ }^{37} \mathrm{H}$. Guillard, C. Viozat, On the behaviour of upwind schemes in the low mach number limit, Computers \& fluids 28 (1) (1999) 63-86.

${ }^{38}$ B. Thornber, D. Drikakis, R. J. Williams, D. Youngs, On entropy generation and dissipation of kinetic energy in highresolution shock-capturing schemes, Journal of Computational Physics 227 (10) (2008) 4853-4872.

${ }^{39} \mathrm{~F}$. Rieper, On the dissipation mechanism of upwind-schemes in the low mach number regime: A comparison between roe and hll, Journal of Computational Physics 229 (2) (2010) 221-232.

${ }^{40} \mathrm{G}$. A. Sod, A survey of several finite difference methods for systems of nonlinear hyperbolic conservation laws, Journal of computational physics 27 (1) (1978) 1-31.

${ }^{41}$ C.-W. Shu, S. Osher, Efficient implementation of essentially nonoscillatory shock-capturing schemes, Journal of computational physics 77 (2) (1988) 439-471.

${ }^{42} \mathrm{C}$. W. Schulz-Rinne, Classification of the riemann problem for two-dimensional gas dynamics, SIAM journal on mathematical analysis 24 (1) (1993) 76-88.

${ }^{43}$ A. Kurganov, E. Tadmor, Solution of two-dimensional riemann problems for gas dynamics without riemann problem solvers, $\mathrm{Nu}-$ merical Methods for Partial Differential Equations: An International Journal 18 (5) (2002) 584-608.

${ }^{44}$ M. Dumbser, O. Zanotti, R. Loubère, S. Diot, A posteriori subcell limiting of the discontinuous galerkin finite element method for hyperbolic conservation laws, Journal of Computational Physics 278 (2014) 47-75.

${ }^{45}$ X. Deng, B. Xie, H. Teng, F. Xiao, High resolution multi-moment finite volume method for supersonic combustion on unstructured grids, Applied Mathematical Modelling 66 (2019) 404-423. 
${ }^{46}$ X. Deng, B. Xie, F. Xiao, H. Teng, New accurate and efficient method for stiff detonation capturing, AIAA Journal 56 (10) (2018) 4024-4038.

${ }^{47}$ P. Yang, H. D. Ng, H. Teng, Z. Jiang, Initiation structure of oblique detonation waves behind conical shocks, Physics of Fluids 29 (8) (2017) 086104.

${ }^{48}$ Y. Zhang, L. Zhou, J. Gong, H. D. Ng, H. Teng, Effects of activation energy on the instability of oblique detonation surfaces with a one-step chemistry model, Physics of Fluids 30 (10) (2018) 106110.

${ }^{49}$ L. I. Sedov, Similarity and dimensional methods in mechanics, CRC press, 1993.

${ }^{50}$ J. Peng, X. Deng, F. Xiao, A direct ale multi-moment finite volume scheme for the compressible euler equations, Communica- tions in Computational Physics 24 (2018) 1300-1325.

${ }^{51}$ S. Prasanna Kumar, B. Patnaik, G. Liu, A skewed kernel approach for the simulation of shocks using sph, International Journal for Numerical Methods in Engineering 111 (4) (2017) 383400 .

${ }^{52} \mathrm{P}$. Woodward, P. Colella, The numerical simulation of twodimensional fluid flow with strong shocks, Journal of computational physics 54 (1) (1984) 115-173.

${ }^{53} \mathrm{Z}$. Xu, C.-W. Shu, Anti-diffusive flux corrections for high order finite difference weno schemes, Journal of Computational Physics 205 (2) (2005) 458-485.

${ }^{54}$ S. Prasanna Kumar, B. Patnaik, A multimass correction for multicomponent fluid flow simulation using smoothed particle hydrodynamics, International Journal for Numerical Methods in Engineering 113 (13) (2018) 1929-1949. 